\title{
The modified Ramsey theorem is not a Gödel sentence
}

\author{
JAAKKO HINTIKKA
}

\begin{abstract}
Gödelian sentences are self-referential first-order sentences in the language of arithmetics. Perhaps the most celebrated one is the sentence which asserts its own unprovability. It is well known that this sentence is neither provable nor refutable in PA (Peano Arithmetics). Some logicians and philosophers have complained that such a sentence is difficult to grasp given its 'meta-theoretical' content and they started to look for undecidable arithmetical statements which have a combinatorial content. One such sentence is a variant of Ramsey's sentence: the ParisHarrington theorem asserts its undecidability. In the present paper I shall argue that such a sentence is not first-order expressible and thereby it does not provide the desired example of a combinatorial, undecidable arithmetical sentence. Instead I shall argue that it is expressible in Independence-friendly (IF) logic.
\end{abstract}

Keywords: Peano Arithmetics, Gödel's Incompleteness theorem, undecidability, Ramsey theorem, IF logic

Mathematicians, logicians and philosophers have been puzzled by Gödel's first incompleteness theorem ever since it was published. What kinds of limitations on our logic does it reveal? If not all arithmetical truths are provable from Peano axioms by means of first-order logic, what additional resources should we resort to in arithmetic and in mathematical reasoning in general?

One way of trying to answer such questions seems to be to see what the Gödelian true but unprovable sentences are like and how their truth can be established. Gödel's own proof is constructive, but the resulting true but unprovable sentences did not turn out to be interesting mathematically and did not suggest any systematic ways of proving stronger results. It seemed therefore highly interesting when Paris and Harrington (1977) [6] discovered a simple 
modification of the Finite Ramsey Theorem in combinatorial mathematics that could be proved by a straightforward non-finitary combinatorial argument but was not possible to prove by means of finite combinatorial methods. This result (it will be called here modified finite Ramsey theorem or MFRT) has been taken to suggest that logical arguments should be supplemented, if not replaced, in the foundations of mathematics by combinatorial reasoning.

An emphasis on combinatorial reasoning may very well be wellplaced. But if so, this recommendation is not by itself a way out of the Gödelian conundrum. For one thing, the Paris-Harrington modification of FRT is not itself the kind of sentence whose existence Gödel proved. The reason is that it is not a sentence that can be formulated in the kind of language that is used in Peano arithmetic and presupposed in Gödel's theorem, that is, in the language of first-order arithmetic. The first purpose of this note is to show its logical status. Once the logic of the modified finite Ramsey theorem is cleared up, it can be seen that it illustrates certain remarkable facts about computability.

The unmodified FRT can be formulated as follows (cf. [8, pp. 363364]):

(1) For all $k, l, m$, there exists $n$ so large that: If $X=\{1,2, \ldots, n\}$ and if $[X]^{k}=C_{1} \cup C_{2} \cup \cdots \cup C_{l}$, then there exists $Y \subseteq X$ such that $[Y] \geq m$ and $[Y]^{k} \subseteq C_{i}$ for some $i \leq l$.

Here $X$ and $Y$ are sets of natural numbers and $i, j, k, l, m, n$ are natural numbers. The cardinality of any $X$ is $[X]$. Also let $N$ be the set of all natural numbers. For any subset $Z$ of $N,[Z]^{k}$ is the set of all (unordered) subsets of $Z$ with $k$ members.

For the purpose of this paper, it suffices to consider the special case known as the party problem. In it a symmetric relation $\mathrm{R}-$ any given symmetric relation - is assumed to be defined on $N$. Also, $l=2, k=2 . C_{1}$ is the set of all the pairs $\langle x, y\rangle$ of numbers such that $R(x, y)$ and $C_{2}$ of the pairs such that $\sim R(x, y)$. If we think of $R(x, y)$ as the relation of knowing each other, then the resulting 'party problem' illustration asks whether you can assume that there is a uniform set $Y$ of $m$ guests simply by inviting large 
enough a number $n$ of guests. The unmodified FRT answers this question affirmatively.

In the MFRT, an extra requirement is imposed on $Y$, viz. that $Y$ 'large' in the sense that

(2) $[Y]>\min _{x}(x \in Y)$.

What is the logical form of this MFRT? The obvious prima facie answer is, assuming a fixed $R$,

(3) $(\forall m)(\exists n)(\forall X)((X=\{1,2, \ldots, n\}) \supset(\exists Y)(([Y] \geq m) \wedge(Y \subseteq$ $\left.\left.\left.X) \wedge\left(\left([Y]^{2} \subseteq C_{1}\right) \vee\left([Y]^{2} \subseteq C_{2}\right)\right) \wedge\left([Y]>\min _{x}(X \in Y)\right)\right)\right)\right)$.

On the face of things, this is not a Gödel sentence. Gödel's incompleteness theorem deals with first-order arithmetic, whereas (3) contains two second-order quantifiers. However, they range over finite subsets of $N$. By means of the technique Gödel used, we can express such existential quantifiers in terms of first-order quantifiers. This takes care of $(\exists Y)$, and it is easily seen that we can similarly deal with $(\forall X)$. This is undoubtedly the basis for thinking that the MFRT is a Gödel sentence.

But this is not the full story. For the MFRT is supposed to hold for any partition $C_{1}, C_{2}, \ldots$ ergo in the case of the party problem for any symmetric relation $R$. Hence there is in effect an additional quantifier $(\forall R)$ in (3). What is more, this additional quantifier matters, because of its relations of dependence and independence of other quantifiers. One important relation is that $(\exists n)$ must be independent of $(\forall R)$ in order for MFRT to be valid. This independence is the main insight of this paper. This dependence is the crucial fact here.

In order to prove the independence, assume that on the contrary $R$ does depend on $n$. Then given $n$ we can define $R$ in such a way that there are no uniform subsets large enough in $X$. From the unmodified FRT and its proof we can see that it takes a set of at least superexponential function of $m$ to express the size required, i.e. the size at which it is necessary that there it has a uniform subset of $m$ numbers (See e.g. [8, p. 363]). Assume that such a subset $X$ of $N$ of superexponential size is given. 
We can choose $X$ as small as possible so that the cardinality of the only uniform subset $Y$ is precisely $m$, i.e. $[Y]=m$. This only requires choosing the relation $R$ in a suitable way. Suppose now that we rename (reorder) the set $X=\{1,2, \ldots,[X]\}$. The result is structure of the same kind as before, but with a different definition of $R$ on $X$, in other words, a new value of $R$. Otherwise, the numbering of the members of $X$ does not enter into the MFRT. In particular, we can re-order the set $X$ in such a way that the members of the uniform subset $Y$ come last in the re-ordered $X$. It is important that we are dealing with a re-numbering of $n=[X]$ elements and hence presuppose intuitively speaking knowing $n$. Since $[X]$ is a superexponential as a function of $m$, we have

(4) $\min _{x}(x \in Y)>([X]-m)>m=[Y]$

But this violates the 'largeness' requirement (2). The counterassumption is hence impossible, and $R$ must not depend on $n$.

But where does the argument leave the MFRT? We know that it is valid. How is it to be expressed in the first place? We have in to amplify (3) by bringing in the quantifier $(\forall R)$ explicitly. The question is what its dependence and independence relations to the other quantifiers in (3) are. Options include the following:

(5) $(\forall m)(\exists n)(\forall R)(---)$

(6) $(\forall m)(\forall R)(\exists n / \forall R)(---)$

(7) $(\forall m)(\forall R)(\exists n)(---)$

Each of these formulas gives rise to a semantical game. The argument just given shows in effect that each strategy of the vertex in the game with (5) is defeated by a suitable strategy by the falsifier. Hence (5) cannot be true and consequently cannot express MFRT.

Also, (7) is weaker than (6) and weaker than the intended force of MFRT. Hence (6) shows the logical form of the theorem.

Accordingly, MFRT involves an irreducibly independent (IF) quantifier. Hence it cannot be a formula of a traditional first-order formula or equivalent to one. And since Gödel is using a received first-order language, MFRT is not a Gödel sentence. 
The irreducibly IF character of MFRT has other interesting consequences and it is related to important theoretical questions. They are discussed in [2] and [3]. From (6) it is seen that $n$ is a function of $m$ only, $n=r(m)$. This function is not general recursive. If it were, it would be definable in terms of a finite set of equations. This set corresponds to a set of traditional first-order sentences. Derivations from these in turn correspond to computations (see [2]). In the case of $f$, the formula (6) can serve as one of these sentences. But it cannot if $f$ is to be general recursive, for (6) involves irreducibly IF Skolem functions. Hence $f$ cannot be general recursive.

Yet $f$ is obviously computable by a mechanical process, for we can simply by going through for a given $m$ all the possible relations $R$ (different 'colorings') for $n=m, m+1, m+2, \ldots$ Hence MFRT appears to be highly interesting even if it is not a Gödel sentence. It is a counterexample to Church's Thesis: in a pretheoretical sense computable, but not general recursive nor therefore a Turing machine computable function.

This line of thought clearly needs fuller argument than what can be given in general. The current research is not free of confusion, and even mistakes, and needs systematic scrutiny (cf. [5]).

These questions are not examined in any detail in this paper, however. Instead, we return briefly to the initial question raised in this paper. It is not a good strategy in trying to understand Gödel's first incompleteness theorem to examine particular instances of true but unprovable arithmetical sentences. What Gödel's theorem says is essentially that the set of true arithmetical sentences is not recursively enumerable. The different axiomatizations are but different methods of enumeration (see [4]). That some particular true arithmetical sentence is not provable in some particular axiomatization is hence informative only of why one particular attempted enumeration fails, not about why every such enumeration fails, that is, what Gödel's incompleteness really means.

\section{References}

[1] Friedman, H., N. Robertson and P. Seymour, The metamathematics of the graph minor theorem, in S. Simpson (ed.), Logic and 
Combinatorics, Contemporary Mathematics, 65, American Mathematical Society, Providence, R.I., 1987, pp. 229-261.

[2] HintikkA, J., Logic as a theory of computability, Newsletter on Philosophy and Computers. APA Newsletters 11(1):2-5, 2011.

[3] Hintikka, J., Function logic and some of its applications, forthcoming.

[4] Hintikka, J., On the significance of incompleteness results. Teorema. Forthcoming.

[5] Hintikka, J., Counter-examples to Kruskal-type theorems, forthcoming.

[6] PAris, J., and L. Harrington, A mathematical incompleteness in Peano arithmetic, in Jon Barwise (ed.), Handbook of Mathematical Logic, North-Holland, Amsterdam, 1977, pp. 1133-1142.

[7] Simpson, S., Unprovable theorems and fast-growing functions, in S. Simpson (ed.), Logic and Combinatorics, Contemporary Mathematics, 65, American Mathematical Society, Providence, R.I., 1987, pp. 354-397.

[8] Simpson, S. (ed.), Logic and Combinatorics, Contemporary Mathematics, 65, American Mathematical Society, Providence, R.I., 1987. 